\title{
Correction of intermittent hypoxia reduces inflammation in obese subjects with obstructive sleep apnea
}

\author{
Sebastio Perrini, ${ }^{1}$ Angelo Cignarelli, ${ }^{1}$ Vitaliano Nicola Quaranta, ${ }^{2}$ Vito Antonio Falcone, ${ }^{2}$ \\ Stella Kounaki, ${ }^{1}$ Stefania Porro, ${ }^{1}$ Alessandro Ciavarella, ${ }^{1}$ Romina Ficarella, ${ }^{1}$ Maria Barbaro, ${ }^{1}$ \\ Valentina Annamaria Genchi, ${ }^{1}$ Pasquale Nigro, ${ }^{1}$ Pierluigi Carratù, ${ }^{2}$ Annalisa Natalicchio,, \\ Luigi Laviola, ${ }^{1}$ Onofrio Resta, ${ }^{2}$ and Francesco Giorgino \\ 'Department of Emergency and Organ Transplantation - Section of Internal Medicine, Endocrinology, Andrology and \\ Metabolic Diseases, and 'Department of Basic Medical Sciences, Neurosciences and Sense Organs - Section of Respiratory \\ Disease, University of Bari Aldo Moro, Bari, Italy.
}

BACKGROUND. In obese subjects with obstructive sleep apnea (OSA), chronic intermittent hypoxia (CIH) may be linked to systemic and adipose tissue inflammation.

METHODS. We obtained abdominal subcutaneous adipose tissue biopsies from OSA and non-OSA obese (BMI > 35) subjects at baseline and after 24 weeks (T1) of weight-loss intervention plus continuous positive airway pressure (c-PAP) or weight-loss intervention alone, respectively. OSA subjects were grouped according to good (therapeutic) or poor (subtherapeutic) adherence to c-PAP.

RESULTS. At baseline, anthropometric and metabolic parameters, serum cytokines, and adipose tissue mRNA levels of obesity-associated chemokines and inflammatory markers were not different in OSA and non-OSA subjects. At $\mathrm{T} 1$, body weight was significantly reduced in all groups. Serum concentrations of IL-2, IL-4, IL-6, MCP-1, PDCF $\beta$, and VEGF $\alpha$ were reduced by therapeutic c-PAP in OSA subjects and remained unaltered in non-OSA and subtherapeutic c-PAP groups. Similarly, adipose tissue mRNA levels of macrophage-specific (CD68, CD36) and ER stress (ATF4, CHOP, ERO-1) gene markers, as well as of IL-6, PDGF $\beta$, and VEGF $\alpha$, were decreased only in the therapeutic c-PAP group.

CONCLUSION. CIH does not represent an additional factor increasing systemic and adipose tissue inflammation in morbid obesity. However, in subjects with OSA, an effective c-PAP therapy improves systemic and obesity-associated inflammatory markers.

FUNDING. Ministero dell'Università e della Ricerca and Progetti di Rilevante Interesse Nazionale.

Conflict of interest: The authors have declared that no conflict of interest exists.

Submitted: April 3, 2017 Accepted: July 25, 2017 Published: September 7, 2017

Reference information: JCI Insight. 2017;2(17):e94379. https://doi.org/10.1172/jici. insight.94379.

\section{Introduction}

Obesity, increased abdominal adiposity, and associated adipose tissue inflammation are linked with an array of health problems, including insulin resistance, type 2 diabetes, dyslipidemia, fatty liver disease, neurodegenerative disorders, and cardiovascular diseases (1). In recent years, several human population studies have suggested that this disease cluster may be fostered by metabolic, inflammatory, cardiovascular, and neurologic abnormalities. Obstructive sleep apnea (OSA) is a common syndrome, occurring in approximately $15 \%$ of the middle-age population and even more frequently in obese subjects; it is characterized by recurrent narrowing and closure of the upper airway, leading to intermittent oxyhemoglobin desaturation, sleep fragmentation, and daytime sleepiness $(2,3)$. Recent studies have suggested that chronic intermittent hypoxia (CIH) may be associated with systemic and adipose tissue inflammation, and this may further increase obesity-related complications $(4,5)$. Levels of circulating inflammatory mediators, such as C-reactive protein (CRP), IL-6, IL-8, and VCAM, have been reported to be higher in otherwise healthy OSA patients compared with obese controls and appear to be related to the severity of the apnea/hypopnea index (AHI) $(5,6)$. Furthermore, at a tissue level, OSA-related repetitive episodes of hypoxia/reoxygenation have been associated with increased production of ROS, promoting local oxidative stress, fibrosis, and immune cell infiltration (2). Converging data point out that adipose tissue depots become hypoxic as tissue mass expands, such that reduced interstitial 
partial oxygen pressure may underpin the initiation and progression of the inflammatory response in obesity (7-9). The enlargement of adipocytes in obesity may exceed the normal oxygen diffusion distance, thus compromising the effective oxygen supply from the vasculature and leading to localized hypoxia (7). Therefore, OSA-associated $\mathrm{CIH}$ in obesity may exacerbate adipose tissue hypoxia and further produce adipose tissue inflammation and dysfunction by increasing macrophage infiltration and activating redox-sensitive transcription factors and ER stress signaling proteins, such as activating transcription factor-4 (ATF4), C/EBP homologous protein (CHOP), and endoplasmic reticulum oxidoreductin (ERO-1) (10).

Continuous positive airway pressure (c-PAP) therapy is an effective treatment for symptomatic OSA. At least 3 months of c-PAP therapy, applied for a minimum of 4 hours nightly, was associated with reduced blood pressure and pulse rate, amelioration of insulin resistance, and decreased levels of inflammatory markers (CRP, TNF- $\alpha$, and IL-6) $(11,12)$. Furthermore, a recent study has reported that weight-loss intervention combined with c-PAP therapy had an incremental effect on reducing insulin resistance and serum triglyceride levels, as compared with c-PAP alone, even though no significant incremental effects on CRP levels were observed (13). However, this study, as well as previous ones assessing the effects of weight loss and c-PAP therapy on cardiometabolic parameters, did not investigate the effects of these interventions on adipose tissue biological endpoints. Furthermore, previous studies on OSA subjects did not control for the effects of diet and obesity per se.

In this study, we have investigated whether therapeutically effective c-PAP combined with a weightloss intervention can improve the systemic and adipose tissue inflammatory/ER stress abnormalities in OSA subjects.

\section{Results}

Baseline characteristics of the study participants. Anthropometric, clinical, and metabolic characteristics of the obese participants at baseline are shown in Table 1. Age, sex, BMI, neck and waist circumference, metabolic and inflammatory parameters, and physical activity were not different among all groups $(P>0.05$; Table 1). However, systolic and diastolic blood pressure levels were higher in the OSA groups treated with c-PAP compared with non-OSA subjects ( $P<0.05$ vs. non-OSA; Table 1$)$. As expected, polysomnographic variables, such as AHI, oxygen desaturation indexes, oxygen saturation below $90 \%$, and the Epworth Sleepiness Scale, were significantly higher in both therapeutic and subtherapeutic OSA as compared with nonOSA subjects $(P<0.05$ vs. non-OSA; Table 1$)$. After 24 weeks of combined intervention, including c-PAP therapy and a weight-loss program, two distinct subgroups of OSA subjects were identified according to adherence to c-PAP therapy, i.e., a therapeutic c-PAP group (c-PAP use for $\geq 4 \mathrm{~h}$ daily) and a subtherapeutic c-PAP group (c-PAP use for $<4 \mathrm{~h}$ daily) (Figure 1 and Table 1). The mean time of c-PAP use was $5.68 \pm$ 0.75 and $2.88 \pm 1.83$ hours per night in the therapeutic and subtherapeutic c-PAP groups, respectively $(P$ $<0.001)$. It should be noted that baseline AHI was significantly higher and other polysomnographic variables were numerically more elevated in the therapeutic compared with the subtherapeutic c-PAP group $(P<0.05$ vs. subtherapeutic; Table 1$)$, even though anthropometric, clinical, metabolic, and inflammatory parameters were similar and not significantly different in the two subgroups of OSA subjects (Table 1).

Assessment of serum levels of cytokines, chemokines, and growth factors was carried out using a multiplex biometrix immunoassay (Bio-Plex Human Cytokine Assay; Bio-Rad). Of the 27 molecules prespecified in the analysis, none were found to be significantly different among OSA therapeutic and subtherapeutic c-PAP groups and non-OSA subjects ( $P>0.05$ vs. non-OSA; Figure 2 and data not shown). Similarly, adipose tissue mRNA levels of genes associated with hypoxia and inflammatory signaling pathways, including ATF4, CHOP, ERO-1, HIF1 $\alpha$, TLR4, pyrin domain-containing 3 (NLRP3), CD68, CD36, IL-6, MCP-1, PDGF $\beta$, VEGF $\alpha$, and GLUT1, did not show any significant difference in the three experimental groups of subjects $(P>0.05$; Figure 3 and Supplemental Figure 1; supplemental material available online with this article; https://doi.org/10.1172/jci.insight.94379DS1).

Effects of weight-loss program and c-PAP therapy on anthropometric, cardiovascular, and metabolic parameters. After 24 weeks of combined intervention, including c-PAP therapy and a weight-loss program, all groups showed a significant decline in body weight and BMI compared with baseline $(P<0.05$ vs. baseline; Table 2$)$; this change was numerically greater in the OSA therapeutic c-PAP group compared with non-OSA and subtherapeutic c-PAP groups, even though these differences were not statistically significant. A numerically greater reduction in the waist circumference was also observed in the OSA therapeutic c-PAP group (Table 2). In addition, neck circumference was statistically reduced only in the OSA therapeutic c-PAP group $(P<0.05$ vs. baseline; Table 2$)$. 
Table 1. Baseline characteristics of the study population

\begin{tabular}{|c|c|c|c|}
\hline Characteristic & Non-OSA & OSA therapeutic & OSA subtherapeutic \\
\hline No. participants & 15 & 16 & 15 \\
\hline Male sex (\%) & 40 & 75 & 56 \\
\hline Systolic blood pressure (mmHg) & $117.1(14.5)$ & $128.8(11)^{A}$ & $123.8(4)^{A}$ \\
\hline Diastolic blood pressure $(\mathrm{mmHg})$ & $72.5(9.6)$ & $82.3(9)^{A}$ & $81.9(1)^{A}$ \\
\hline HR (bpm) & 73.9 (11.9) & $75.5(10)$ & $75.8(2)$ \\
\hline Weight (kg) & $109.8(18.4)$ & $115.2(18)$ & $108.5(6)$ \\
\hline $\mathrm{BMI}$ & $41.1(9.2)$ & $38(6)$ & $38.7(2)$ \\
\hline Neck (cm) & $40.6(3.1)$ & $43.5(2)$ & $42.2(1)$ \\
\hline \multicolumn{4}{|l|}{ Cholesterol } \\
\hline Total (mg/dl) & $186.6(43.8)$ & 193 (39.2) & $191.3(8.1)$ \\
\hline Fasting insulinemia (mUl/ml) & $26.1(14.5)$ & $30.2(12.2)$ & $25.7(2.7)$ \\
\hline HOMA-IR & $6(3.6)$ & $7.5(3.1)$ & $6.4(1.6)$ \\
\hline Uric acid (mg/dl) & $5.2(1.8)$ & $6.1(2.2)$ & $5.8(1.4)$ \\
\hline Triglycerides (mg/dl) & 119.6 (83.9) & $157.5(53.3)$ & $131.4(13.5)$ \\
\hline AST (UI/I) & $30.2(16.3)$ & $27.9(9.7)$ & $22.7(2.6)$ \\
\hline ALT (UI/I) & $55.9(45.2)$ & $58.2(39.6)$ & $44.4(4.8)$ \\
\hline WBC $\left(n \times 10^{3} / \mathrm{ml}\right)$ & $6.9(2.3)$ & $7.6(1.4)$ & $7.3(1.0)$ \\
\hline Hematocrit (\%) & $41.9(3.1)$ & $44(3.3)$ & $42.6(1.9)$ \\
\hline CRP (mg/dl) & $9.7(9.1)$ & $5.8(6.7)$ & $7.9(2.3)$ \\
\hline $\operatorname{ESR}(\mathrm{mm} / \mathrm{h})$ & $24.1(21.7)$ & $16.9(18.5)$ & $17.3(3.5)$ \\
\hline Total METs (METs/wk) & 2,363.9 (1,939.2) & $1,683(2,757.6)$ & $2,529(4,556.9)$ \\
\hline $\mathrm{ST}_{90}(\%)$ & $1.9(1.7)$ & $34.2(26.4)^{A}$ & $10.8(13.6)^{A}$ \\
\hline $\mathrm{pH}$ & $7.4(0.01)$ & $7.4(0.02)$ & $7.4(0.02)$ \\
\hline $\mathrm{PaO}_{2}$ & $82.3(10.3)$ & $76.4(10.6)$ & 79.5 (9.4) \\
\hline $\mathrm{PaCO}_{2}$ & $40.1(1.9)$ & $41.7(2.8)$ & $40.4(2.8)$ \\
\hline $\mathrm{SaO}_{2}$ & $95.5(1.9)$ & $95.3(1.6)$ & $95.7(1.2)$ \\
\hline $\mathrm{HCO}_{3}$ & $26.3(0.7)$ & $28.3(2.8)$ & $27.1(2.3)$ \\
\hline
\end{tabular}

Values shown throughout the table are mean (SD). ${ }^{A} P<0.05$ vs. non-OSA (1-way ANOVA); ${ }^{B} P<0.05$ vs. OSA subtherapeutic (1-way ANOVA). AHI, apnea/ hypopnea index; CRP, C-reactive protein; ESR, erythrocyte sedimentation rate; ESS, Epworth Sleepiness Scale; HR, heart rate; MET, metabolic equivalent of the task; ODI, oxygen desaturation index; $\mathrm{ST}_{90}$, total sleep time spent with $\mathrm{SaO}_{2}<90 \%$.

Furthermore, 24 weeks of the specific intervention resulted in significant reductions in systolic blood pressure, HOMA-IR, aspartate aminotransferase (AST), and uric acid in the OSA therapeutic c-PAP group ( $P<0.05$ vs. baseline; Table 2$)$. There was a greater decline in systolic and diastolic blood pressure, as well as in AST, in the OSA therapeutic c-PAP group compared with that in non-OSA and OSA subtherapeutic c-PAP groups ( $P<0.05$ vs. non-OSA and OSA subtherapeutic c-PAP; Table 2$)$. By contrast, when compared with baseline, heart rate was significantly reduced only in non-OSA and OSA subtherapeutic c-PAP groups and nonsignificantly reduced in the OSA therapeutic c-PAP group $(P<0.05$ vs. baseline; Table 2). Following intervention, there were no significant changes in total and LDL cholesterol, 

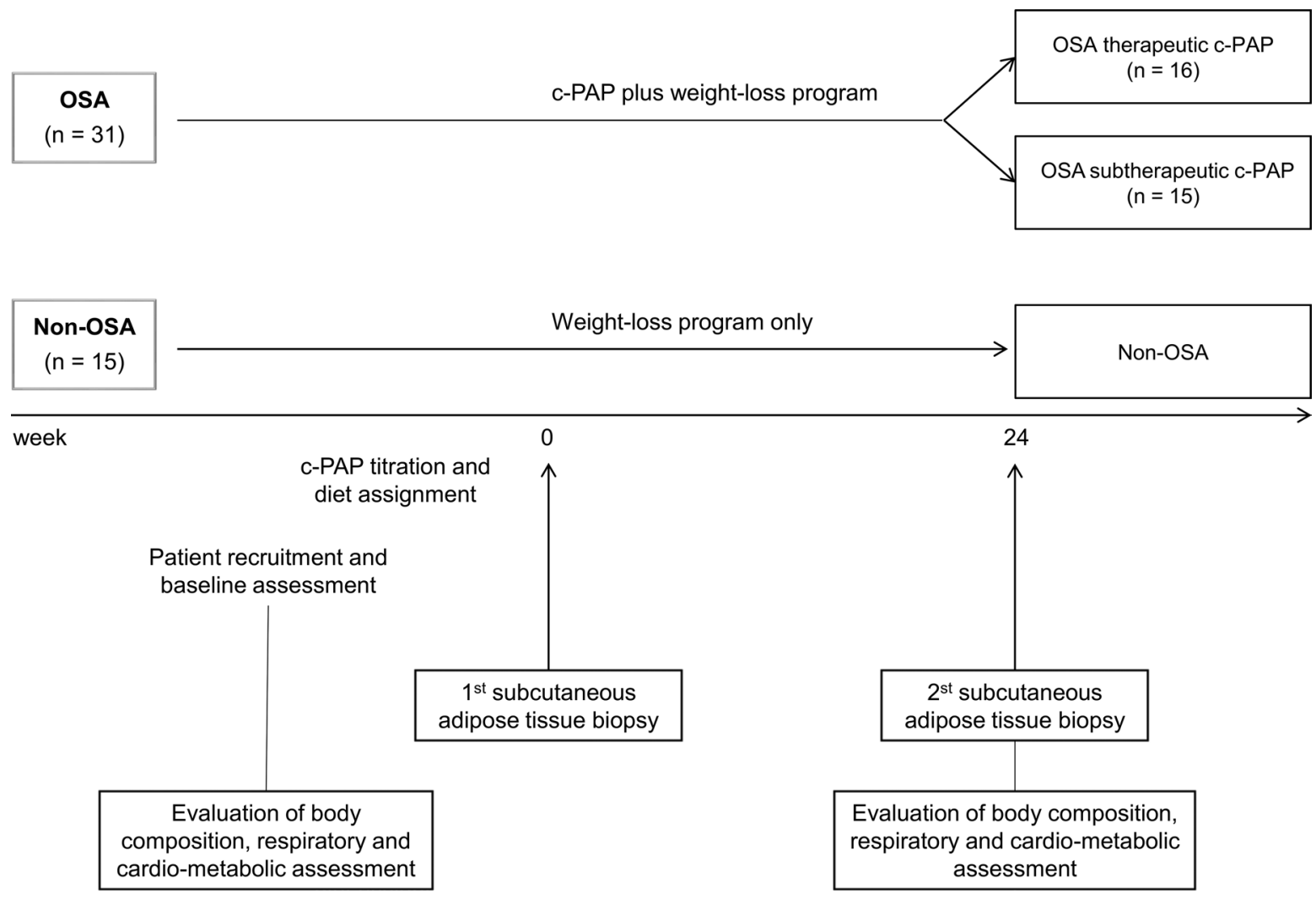

Figure 1. Study design. OSA, obstructive sleep apnea; c-PAP, continuous positive airway pressure.

triglycerides, fasting glycemia and insulinemia, HbA1c, alanine aminotransferase (ALT), and physical activity in the three groups, even though there were apparently numerically greater reductions in total and LDL cholesterol and ALT levels in OSA therapeutic c-PAP subjects compared with non-OSA and OSA subtherapeutic c-PAP subjects $(P>0.05$; Table 2$)$.

Effects of weight-loss program and c-PAP on systemic and adipose tissue inflammatory markers. Circulating general inflammatory markers, such as white blood cell (WBC) count, CRP, erythrocyte sedimentation rate (ESR), and fibrinogen, did not show any significant changes following the specific intervention in all experimental groups $(P>0.05$; Table 2$)$. In contrast, of the 27 serum cytokines assessed, IL-2, IL-4, MCP-1, PDGF $\beta$, and VEGF $\alpha$ were found to be significantly reduced compared with baseline in the OSA therapeutic c-PAP group but not in the non-OSA and OSA subtherapeutic c-PAP groups $(P<0.05$ vs. baseline; Figure 4). Furthermore, changes in serum levels of IL-4, PDGF $\beta$, and VEGF $\alpha$ in OSA subjects who underwent therapeutic c-PAP were also significantly different compared with those occurring in non-OSA and subtherapeutic c-PAP groups ( $P<0.05$ vs. non-OSA and subtherapeutic c-PAP; Figure 4). Serum levels of IL- 6 were significantly reduced compared with baseline in non-OSA and in OSA therapeutic c-PAP subjects $(P<0.05$ vs. baseline) but not in the OSA subtherapeutic c-PAP group (Figure 4).

In line with the results on circulating cytokines and growth factors, correction of $\mathrm{CIH}$ with therapeutic c-PAP in OSA subjects induced significant reductions in adipose tissue mRNA levels of ATF4, CHOP, ERO-1, CD68, CD36, IL-6, PDGF $\beta$, and VEGF $\alpha$ ( $P<0.05$ vs. baseline; Figure 5), whereas no change was observed in non-OSA and OSA subtherapeutic c-PAP subjects $(P<0.05$ vs. non-OSA and subtherapeutic c-PAP; Figure 5). Furthermore, the changes in ERO-1, CD68, and CD36 mRNA levels observed in OSA therapeutic c-PAP subjects were significantly different compared with those of the non-OSA and subtherapeutic c-PAP groups $(P$ $<0.05$ vs. non-OSA and subtherapeutic c-PAP; Figure 5). There were no significant changes in HIF1 $\alpha$, TLR4, NLRP3, and GLUT1 in the experimental groups, even though there was a nonsignificant trend for reduction of these factors in the OSA subjects who underwent therapeutic c-PAP (Figure 5 and Supplemental Figure 1). In addition, among those cytokines and genes that were significantly reduced in the OSA therapeutic c-PAP group compared with OSA subtherapeutic c-PAP and non-OSA groups, there was a negative relationship with 

IL-2
IL-4
IL-10
IL-6
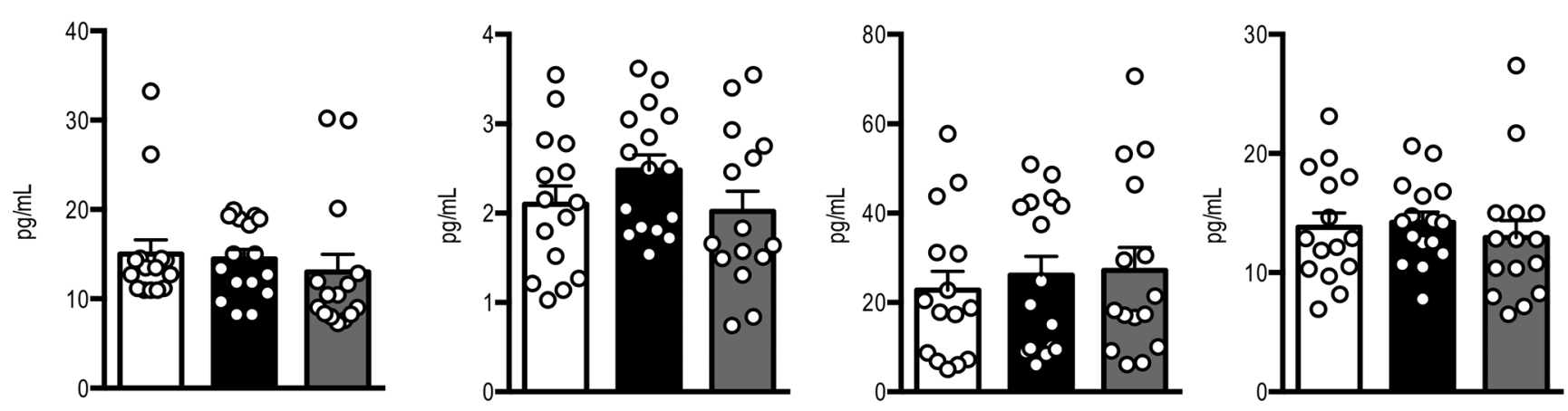

MCP-1

PDGF $\beta$

VEGFa

RANTES
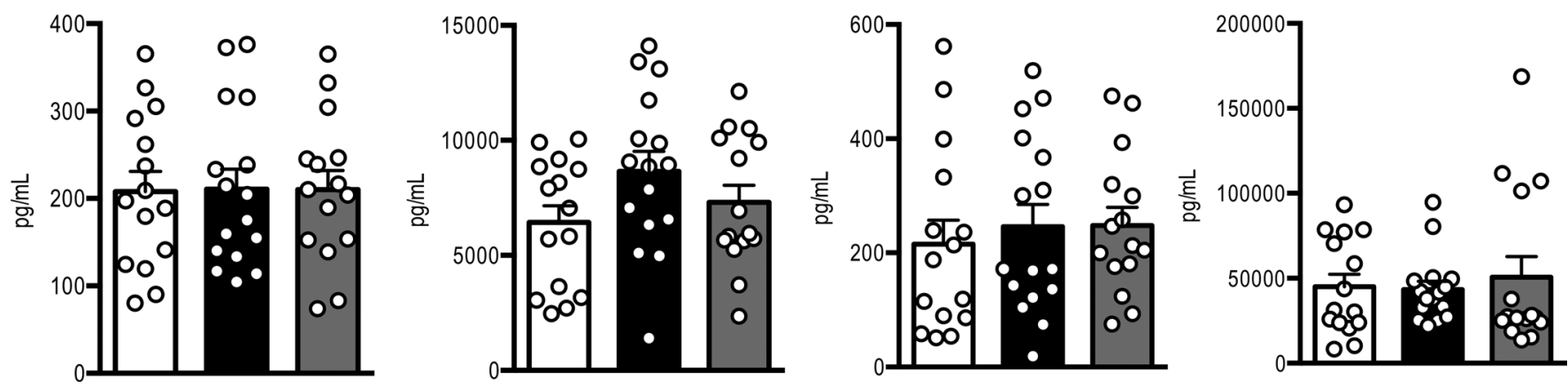

Figure 2. Baseline serum cytokine concentrations in non-OSA, OSA therapeutic c-PAP, and OSA subtherapeutic c-PAP obese subjects. Circulating levels were assessed in non-OSA (white bars), OSA therapeutic c-PAP (black bars), and OSA subtherapeutic c-PAP (gray bars) obese subjects at baseline. Serum levels $(\mathrm{pg} / \mathrm{ml})$ were determined by the Bio-Plex assay, as described in the Methods. Results are mean \pm SEM $(n=2$ measurements for each subject; $n=15$ non-OSA, $n=16$ OSA therapeutic c-PAP; and $n=15$ OSA subtherapeutic c-PAP). Differences among groups were assessed with 1-way ANOVA statistical analysis. OSA, obstructive sleep apnea; c-PAP, continuous positive airway pressure.

time of c-PAP use (Figure $6 ; P<0.05)$. Specifically, a more prolonged c-PAP use was negatively correlated with significant reductions of IL-4 $(P=0.041 ; \mathrm{r}=-0.322)$ and VEGF $\alpha(P=0.0001 ; \mathrm{r}=-0.322)$ as well as adipose tissue mRNA levels of CD36 $(P=0.001 ; \mathrm{r}=-0.578), \operatorname{CD} 68(P=0.004 ; \mathrm{r}=-0.500)$, and ERO-1 $(P=0.002$; $\mathrm{r}=-0.543)$ (Figure 6). There was also a nonsignificant trend for reduction of PDGF $\beta(P=0.113 ; \mathrm{r}=-0.290$; Figure 6). Finally, no significant correlations were found between the Epworth Sleepiness Scale and mRNA levels of CD36, CD68, and ERO-1 at baseline, with nonsignificant trends for correlation between reductions in these parameters observed after 24 weeks of therapeutic c-PAP (Supplemental Figure 2).

\section{Discussion}

In morbidly obese individuals with OSA, we show that correction of CIH by an effective c-PAP therapy combined with a weight-loss intervention program resulted in reduced plasma levels of some proinflammatory cytokines and growth factors, such as IL-2, IL-4, IL-6, MCP-1, PDGF $\beta$, and VEGF $\alpha$, as well as in diminished mRNA expression of markers related to tissue hypoxia, ER stress, inflammation, and macrophage infiltration, such as ATF4, CHOP, ERO-1, CD68, CD36, IL-6, PDGF $\beta$, and VEGF $\alpha$, in adipose tissue. These changes did not occur, or occurred only minimally, in obese OSA subjects in which c-PAP was not effective in correcting CIH. In addition, they also were not apparent in control non-OSA subjects treated with the weight-loss intervention program alone.

A direct link among adipose cell hypertrophy, hypoxia, and inflammatory response has been long suggested. Adipocyte hypertrophy occurring during adipose tissue expansion may increase cell diameter to a level exceeding the diffusion limit of oxygen, thus creating areas of local adipose tissue microhypoxia that, 


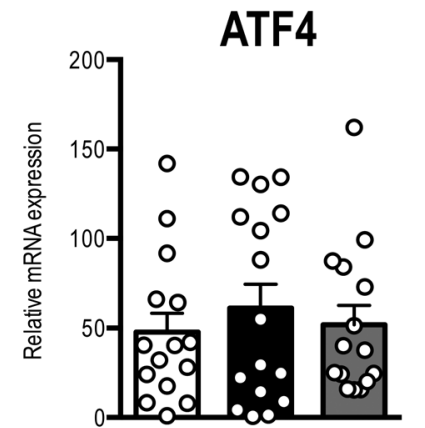

TLR4

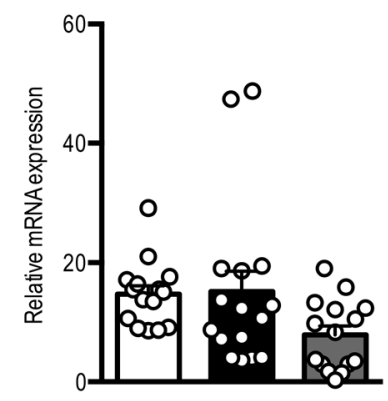

IL-6

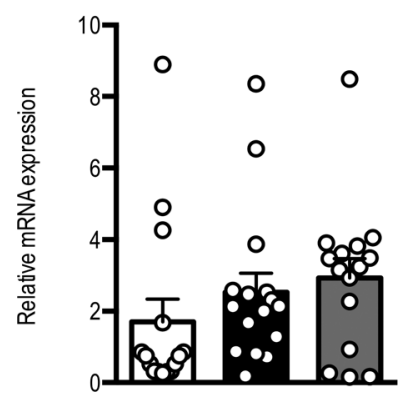

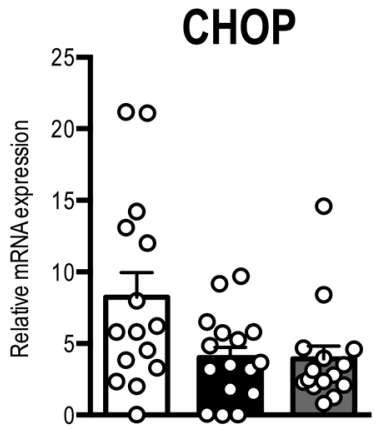

NLRP3

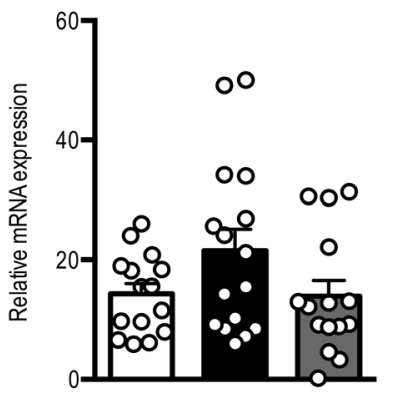

MCP-1

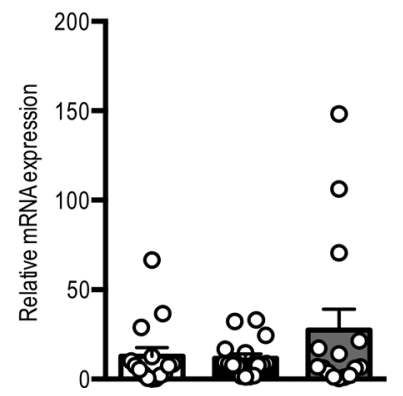

ERO-1

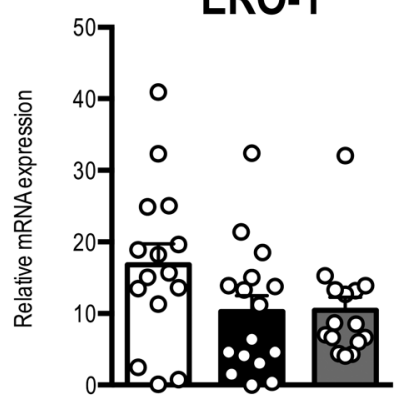

CD68

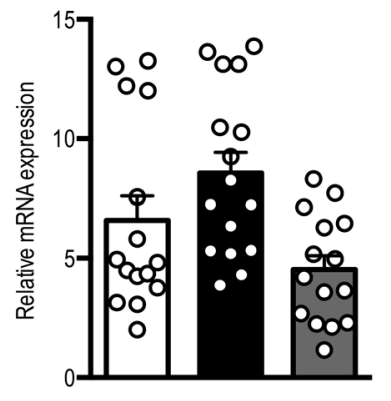

PDGF $\beta$

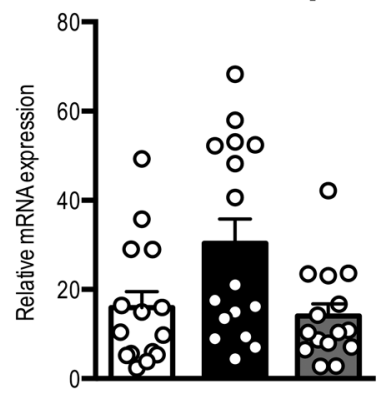

HIF1a

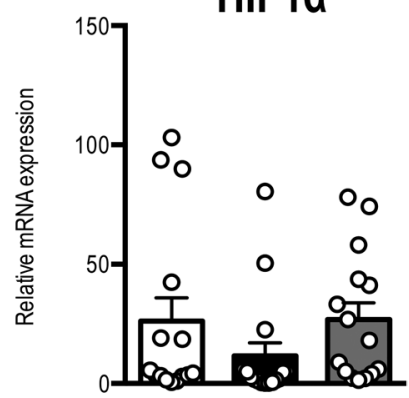

CD36

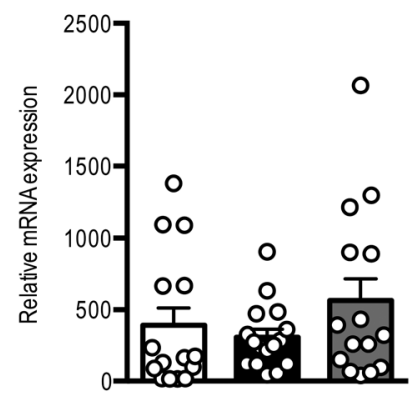

VEGFa

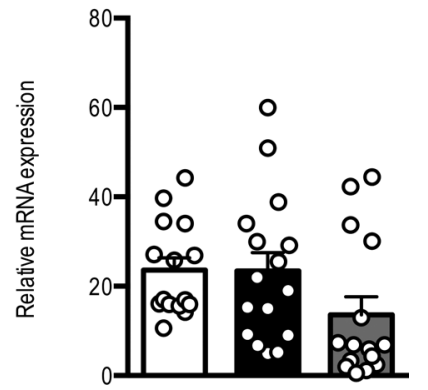

Figure 3. Baseline mRNA expression of ER stress and inflammatory genes in subcutaneous adipose tissue from non-OSA, OSA therapeutic c-PAP, and OSA subtherapeutic c-PAP obese subjects. Subcutaneous adipose tissue biopsies were obtained from non-OSA (white bars), OSA therapeutic c-PAP (black bars), and OSA subtherapeutic c-PAP (gray bars) obese subjects at baseline. mRNA levels of ER stress and inflammatory genes were determined by quantitative RT-PCR and normalized to $18 \mathrm{~S}$ rRNA. Results are mean \pm SEM of values in adipose tissue ( $n=2$ measurements for each subject; $n=15$ non-OSA; $n=16$ OSA therapeutic c-PAP; and $n=15$ OSA subtherapeutic c-PAP). Differences among groups were assessed with 1-way ANOVA statistical analysis. ER, endoplasmic reticulum; OSA, obstructive sleep apnea; c-PAP, continuous positive airway pressure.

in turn, may initiate and/or enhance secretion of proinflammatory cytokines as well as dysregulated fatty acid fluxes and adipocyte death (14). In obese mice, areas of hypoxia overlapped to a large extent with the presence of macrophages, indicating that the expansion of adipose tissue, resulting from combined hypertrophy and hyperplasia, is associated with low $\mathrm{pO}_{2}$ and accumulation of immune cells (15). In humans, the capillary density and tissue oxygen tension were found to be lower in overweight/obese subjects than in lean subjects in both visceral and subcutaneous adipose tissues (16-18). As shown by Goossens et al., obese insulin-resistant individuals exhibited lower adipose tissue blood flow but higher adipose tissue $\mathrm{pO}_{2}$, which could be explained by lower oxygen consumption by the adipose tissue (18). Furthermore, impaired adipose tissue $\mathrm{pO}_{2}$ was paralleled by increased expression and secretion of adipokines and markers of macrophage infiltration (8). In addition, in morbidly obese patients, $\mathrm{CIH}$ was shown to be associated with more severe liver injuries, but it did not worsen obesity-induced macrophage accumulation in both subcutaneous and visceral adipose tissue (19). Similarly, in our study, the presence of CIH did not further augment serum proinflammatory cytokines and growth factors or adipose tissue ER stress and hypoxia markers as compared with BMI-matched obesity without CIH (Figures 2 and 3). A potential explana- 
Table 2. Changes from baseline in anthropometric and metabolic variables in the study population after 24 weeks of intervention

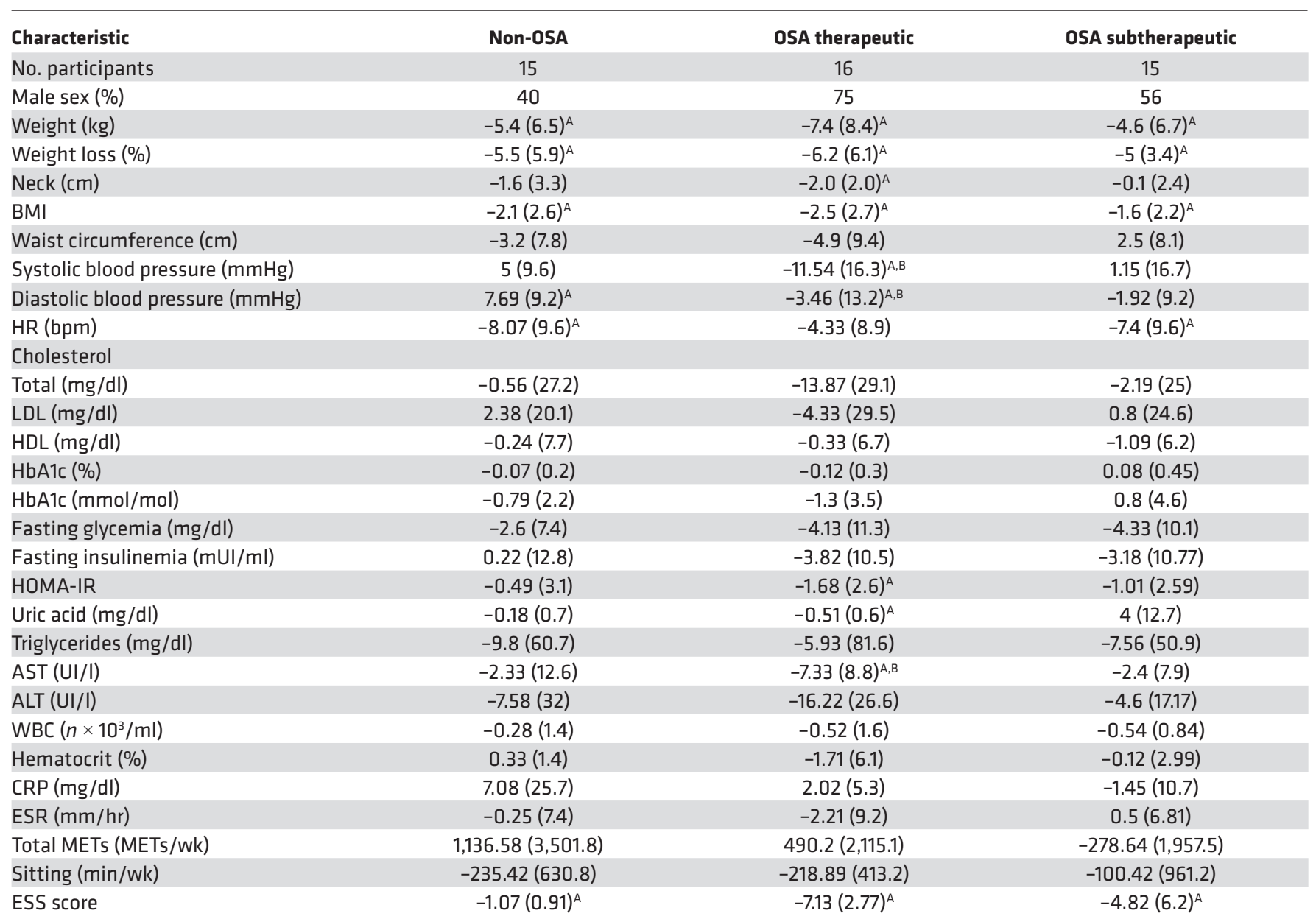

Values shown throughout the table are mean (SD). ${ }^{A} P<0.05$ vs. baseline (1-sample $t$ test); ${ }^{B} P<0.05$ vs. non-OSA and OSA subtherapeutic (1-way ANOVA). CRP, C-reactive protein; ESR, erythrocyte sedimentation rate; HR, heart rate; MET, metabolic equivalent of the task.

tion could be that, as adipose tissue massively expands over its vascular capacity when obesity exceeds a BMI of 35, a high grade of local and systemic inflammation is reached that is not further worsened by OSA-induced CIH (Figure 7). Interestingly, in obese C57BL/6 mice, obesity-associated increases in leptin and TNF- $\alpha$ overwhelmed the effects of experimentally induced hypoxia on these markers (20). It is to be mentioned that another study in morbidly obese patients found higher serum TNF- $\alpha$ and ICAM- 1 and greater TNF- $\alpha$ immunostaining in gluteal subcutaneous adipose tissue with increasing AHI (5). However, these subjects also had type 2 diabetes, which may impair TNF- $\alpha$ serum levels independently of measures of adiposity (21); in addition, the subcutaneous adipose tissue biopsies were obtained only from a subset of individuals, and this may have generated a selection bias (5).

After 24 weeks of intervention, each group of subjects showed significant reductions in body weight and BMI as compared with baseline. However, significant improvements of insulin resistance, measured by HOMA-IR, and more prominent decreases in blood pressure and AST were observed only in OSA subjects, in which the c-PAP combined with weight-loss regimen achieved correction of $\mathrm{CIH}$. Even though no significant changes in systemic inflammatory markers (WBC count, CRP, ESR, and fibrinogen) were seen, this was associated with more evident decreases in circulating cytokines and growth factors and adipose tissue gene markers of ER stress, macrophage infiltration, and angiogenic cytokines. Previous studies (13, 22) have shown that therapeutic c-PAP has significant ameliorative effects on insulin resistance and induces larger reductions in weight, BMI, and systolic blood pressure compared with weight-loss intervention alone. However, in one study (13), therapeutic c-PAP had no effects on general systemic inflammation 
IL-2

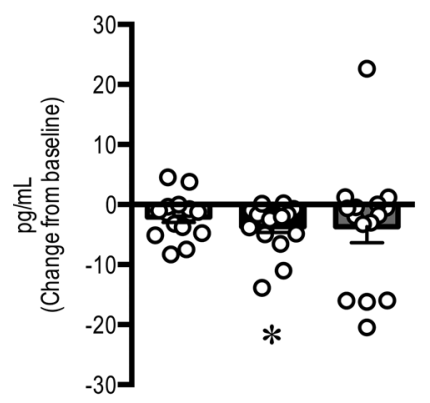

MCP-1

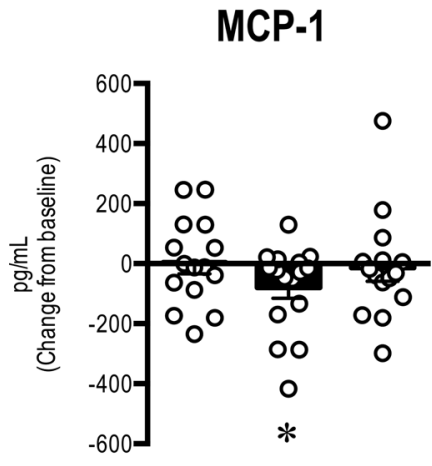

IL-4

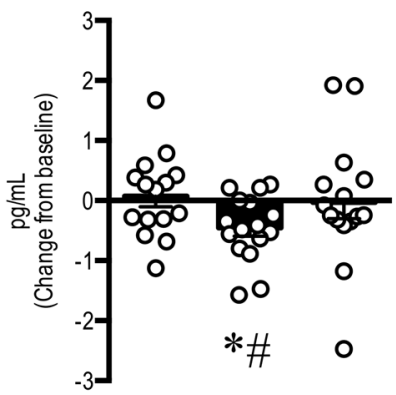

PDGF $\beta$

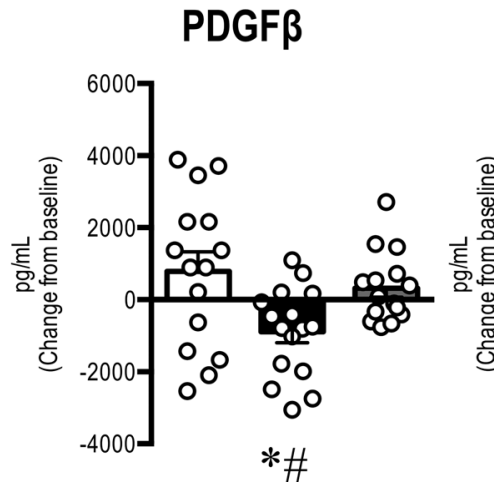

IL-6

IL-10
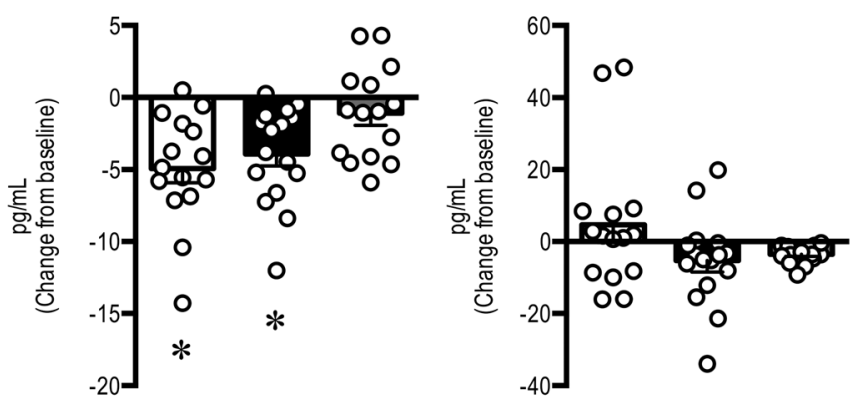

Figure 4. Effects of intervention on circulating cytokines. Serum levels of IL-2, IL-4, IL-6, IL-10, MCP-1, PDGF $\beta$, VEGF $\alpha$, and RANTES were assessed in non-OSA (white bars), OSA therapeutic c-PAP (black bars), and OSA subtherapeutic c-PAP (gray bars) subjects before and after 24 weeks of treatment, as indicated in Figure 1. Absolute serum levels $(\mathrm{pg} / \mathrm{ml})$ were determined by Bio-Plex assay, as described in the Methods. Results shown are mean \pm SEM of changes versus baseline ( $n=2$ measurements for each subject; $n=15$ non-0SA; $n=16$ OSA therapeutic c-PAP; and $n=15$ OSA subtherapeutic c-PAP). ${ }^{*} P<0.05$ vs. baseline (1-sample $t$ test); ${ }^{\#} P<0.05$ vs. non-OSA and OSA subtherapeutic c-PAP (1-way ANOVA). OSA, obstructive sleep apnea; c-PAP, continuous positive airway pressure.

parameters, such as WBC count, CRP, ESR, and fibrinogen. Indeed, several cross-sectional and prospective observational studies have yielded conflicting results on the association between CRP and sleep-disordered breathing as well as on the effect of c-PAP therapy on CRP levels (13, 23-26). Similarly to our results, in a double-blind randomized controlled trial comparing therapeutic and subtherapeutic c-PAP, effective correction of $\mathrm{CIH}$ in obese subjects with moderate-to-severe OSA did not significantly change CRP levels from baseline (27). Other large studies also showed no significant improvement in CRP levels after 6 to 12 months of c-PAP therapy in patients with $\operatorname{OSA}(13,23)$.

In contrast to CRP, circulating levels of IL-2, IL-4, IL-6, MCP-1, PDGF $\beta$, and VEGF $\alpha$, which represent sensitive serum markers of low-grade inflammation and/or are more closely related to changes in adipose tissue mass and characteristics, were significantly reduced by effective c-PAP therapy. For IL-4, PDGF $\beta$, and VEGF $\alpha$, changes were apparently opposite in therapeutic c-PAP compared with those occurring in non-OSA and subtherapeutic c-PAP groups (Figure 4). This is in line with previously published meta-analyses showing that c-PAP therapy significantly decreases the levels of cytokines and chemokines, such as IL-6, IL-8, and TNF- $\alpha$ in OSA patients, albeit with some differences among the various inflammatory markers (12). The angiogenic factors PDGF $\beta$ and VEGF $\alpha$ are potent chemoattractants and mitogens for vascular smooth cells, and several studies have correlated their serum levels with the degree of hypoxia and severity of peripheral artery disease (28-30). For the first time to our knowledge, we show that effective c-PAP therapy lowers serum levels as well as adipose tissue mRNA expression of PDGF $\beta$ and VEGF $\alpha$, and this may be potentially relevant for counteracting the development of atherosclerosis. Furthermore, correction of $\mathrm{CIH}$ by therapeutic c-PAP reduced serum concentrations of the chemotactic factor MCP-1, which correlates with the extent of adipose tissue hypoxia and macrophage infiltration, insulin resistance, and hepatic steatosis in both mouse and human obesity (31-33). Importantly, adipose tissue mRNA levels of macrophage-specific markers, such as CD68 and CD36, were also significantly reduced by therapeutic 

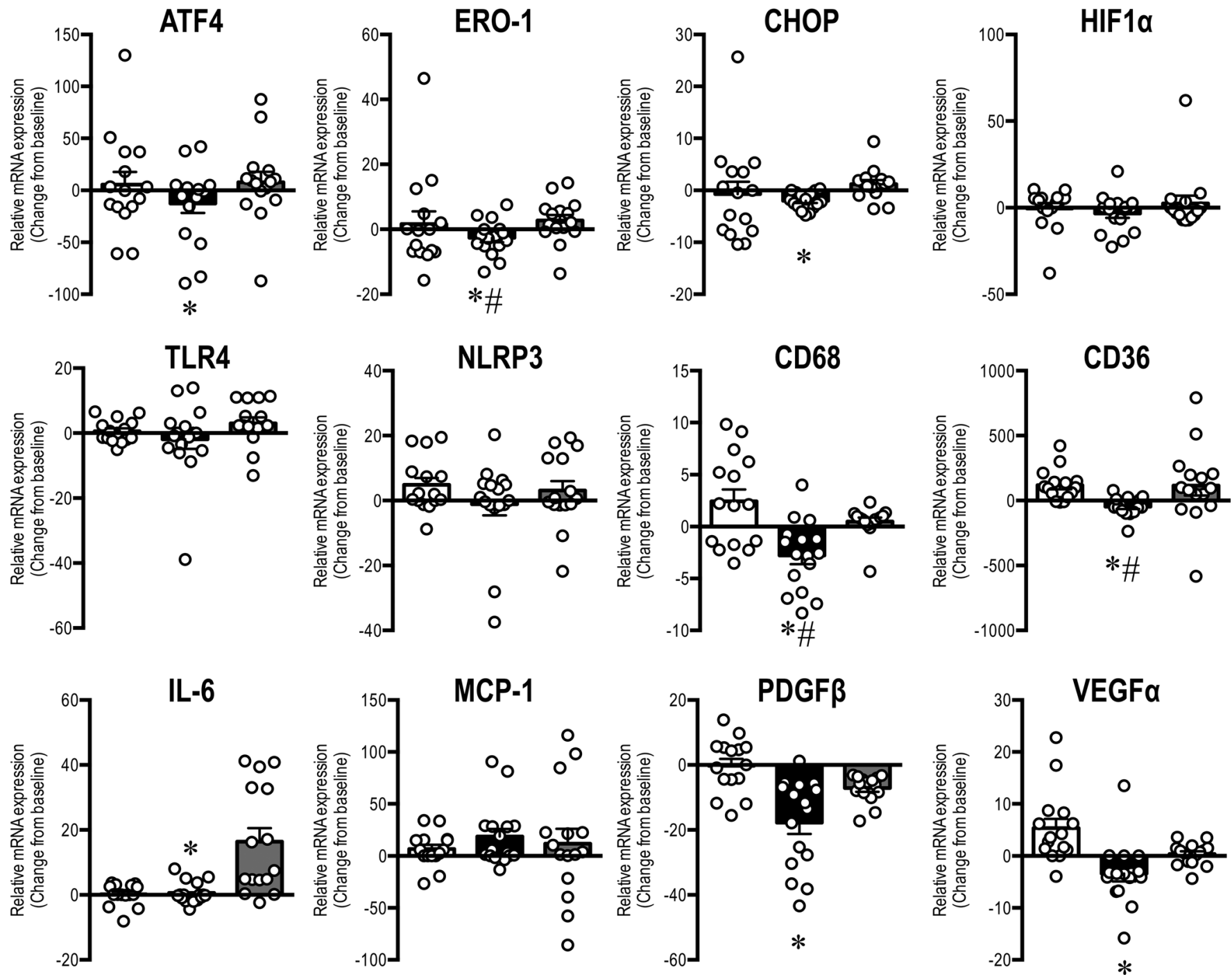

Figure 5. Effects of intervention on mRNA expression of ER stress and inflammatory genes in subcutaneous adipose tissue. Subcutaneous adipose tissue biopsies were obtained in non-OSA (white bars), OSA therapeutic c-PAP (black bars), and OSA subtherapeutic c-PAP (gray bars) subjects before and after 24 weeks of treatment, as indicated in Figure 1. mRNA levels of ER stress and inflammatory genes were determined by quantitative RT-PCR and normalized to $18 \mathrm{~S}$ rRNA. Results are mean \pm SEM of changes versus baseline ( $n=2$ measurements for each subject; $n=15$ non-0SA; $n=16$ OSA therapeutic c-PAP; and $n=15$ OSA subtherapeutic c-PAP). ${ }^{*} P<0.05$ vs. baseline (1-sample $t$ test). $\# P<0.05$ vs. non-OSA and OSA subtherapeutic c-PAP (1-way ANOVA). ER, endoplasmic reticulum; OSA, obstructive sleep apnea; c-PAP, continuous positive airway pressure.

c-PAP in OSA subjects (Figure 5). These results are of interest in light of the observations that inhibition of MCP-1 was shown to improve insulin resistance and reduce macrophage infiltration into adipose tissue of obese mice (32) and that expression of both CD68 and CD36 is increased in adipose tissue in obesity and correlates with reduced levels of adipose tissue $\mathrm{pO}_{2}$, increased macrophage infiltration, and tissue inflammation (34-37). Furthermore, CD36 deficiency protected mice from chronic adipose tissue inflammation induced by high-fat diet feeding (38).

ER stress-related genes known to be modulated by hypoxia and high-grade systemic and adipose tissue inflammation, such as ATF4, CHOP, and ERO-1, were also found to be significantly and specifically reduced by therapeutic c-PAP in this study (Figure 5). An aberrant ER stress pathway and the related signaling networks correlate with adipose tissue inflammation and chronic metabolic diseases, such as obesity, insulin resistance, and type 2 diabetes. Activation of ER stress response results in increased mRNA levels of ATF4, and this leads to expression of genes involved in apoptosis, as well as of CHOP and ERO-1 (10). The causality between impaired ER stress signaling and increased systemic inflammation is supported by experimental evidence, since ER stress induction was previously shown to lead to increased expression of 


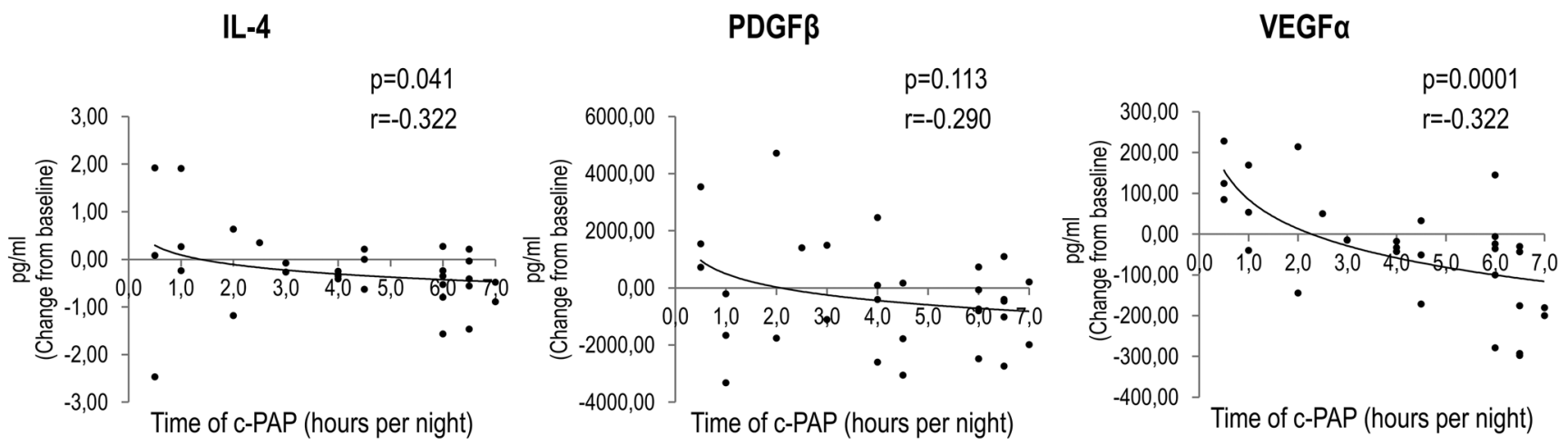

CD36

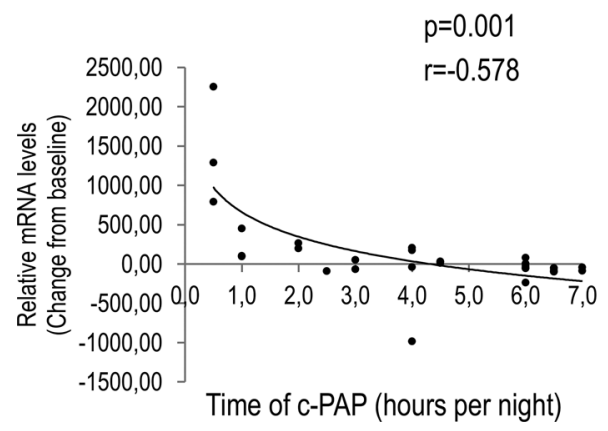

CD68

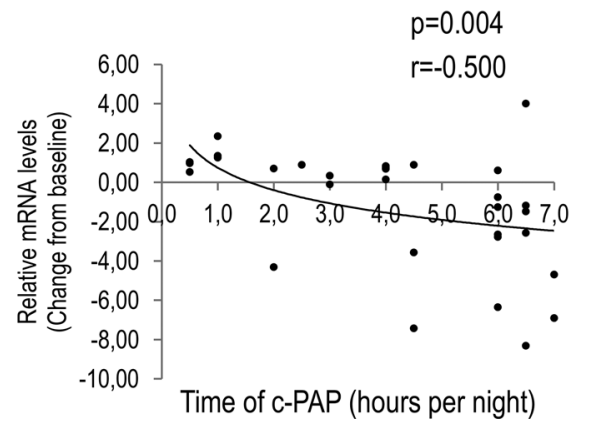

ERO-1

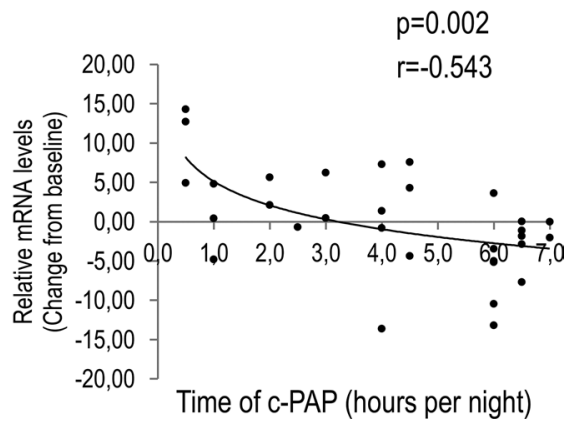

Figure 6. Relationship between time of c-PAP use and systemic and adipose tissue inflammation. Correlation between time of c-PAP use and serum levels of IL-4, PDGF $\beta$, and VEGF $\alpha$ in OSA obese subjects (top). Correlation between time of c-PAP use and adipose tissue mRNA levels of CD36, CD68, and ER01 in OSA obese subjects (bottom) ( $n=16$ OSA therapeutic c-PAP subjects; $n=15$ OSA subtherapeutic c-PAP subjects). Statistical analysis was performed using Pearson correlation. OSA, obstructive sleep apnea; c-PAP, continuous positive airway pressure.

proinflammatory molecules, such as IL-8, IL-6, MCP-1, and TNF- $\alpha$ (39). These findings are in line with our results of coordinate reduction of ATF4, CHOP, and ERO-1 mRNA levels and of proinflammatory cytokines, such as MCP-1, IL-6, and IL-2, in OSA subjects undergoing a therapeutic c-PAP with CIH correction. Recently, a link between the ER stress pathway and NLRP3 inflammasome-related genes has been suggested (40-42). A nonsignificant trend for reduction of TLR4 and NLRP3 mRNA expression in adipose tissue was observed in the OSA therapeutic c-PAP subjects in this study.

Moderate weight loss in non-OSA subjects did not bring about any decline of systemic and adipose tissue markers of inflammation. This is in line with a recent report showing that, in obese subjects, a $5 \%$ weight loss improved adipose tissue, liver, and muscle insulin sensitivity, as well as $\beta$ cell function, without a concomitant change in systemic or subcutaneous adipose tissue markers of inflammation (43).

In conclusion, $\mathrm{CIH}$ does not represent an additional factor increasing systemic and adipose tissue inflammation in individuals with class II and class III obesity. However, weight-loss intervention combined with c-PAP therapy significantly improves the inflammatory profile of these obese subjects with OSA, in which correcting nocturnal oxygen desaturation is associated with reduced serum levels of proinflammatory cytokines and mRNA levels of gene markers related to ER stress, macrophage infiltration, and inflammation (Figure 7). Thus, effective c-PAP therapy associated with weight loss has the potential to reduce cardiometabolic risk in severe obesity associated with OSA.

Limitations and strengths of the study. A limitation of the current study is that we measured only mRNA and not protein levels of ER stress/inflammation marker genes in adipose tissue. Similar to what was recently reported by Kursawe et al. (44), this was due to the limited amount of subcutaneous adipose tissue obtained from the enrolled subjects. Furthermore, we did not assess the visceral adipose tissue and thus cannot rule out that the results obtained in abdominal subcutaneous adipose tissue could be different in the visceral fat depot. However, other studies have shown that in morbidly obese subjects the levels of inflammation measured by evaluating macrophage accumulation were of similar magnitude for both visceral 


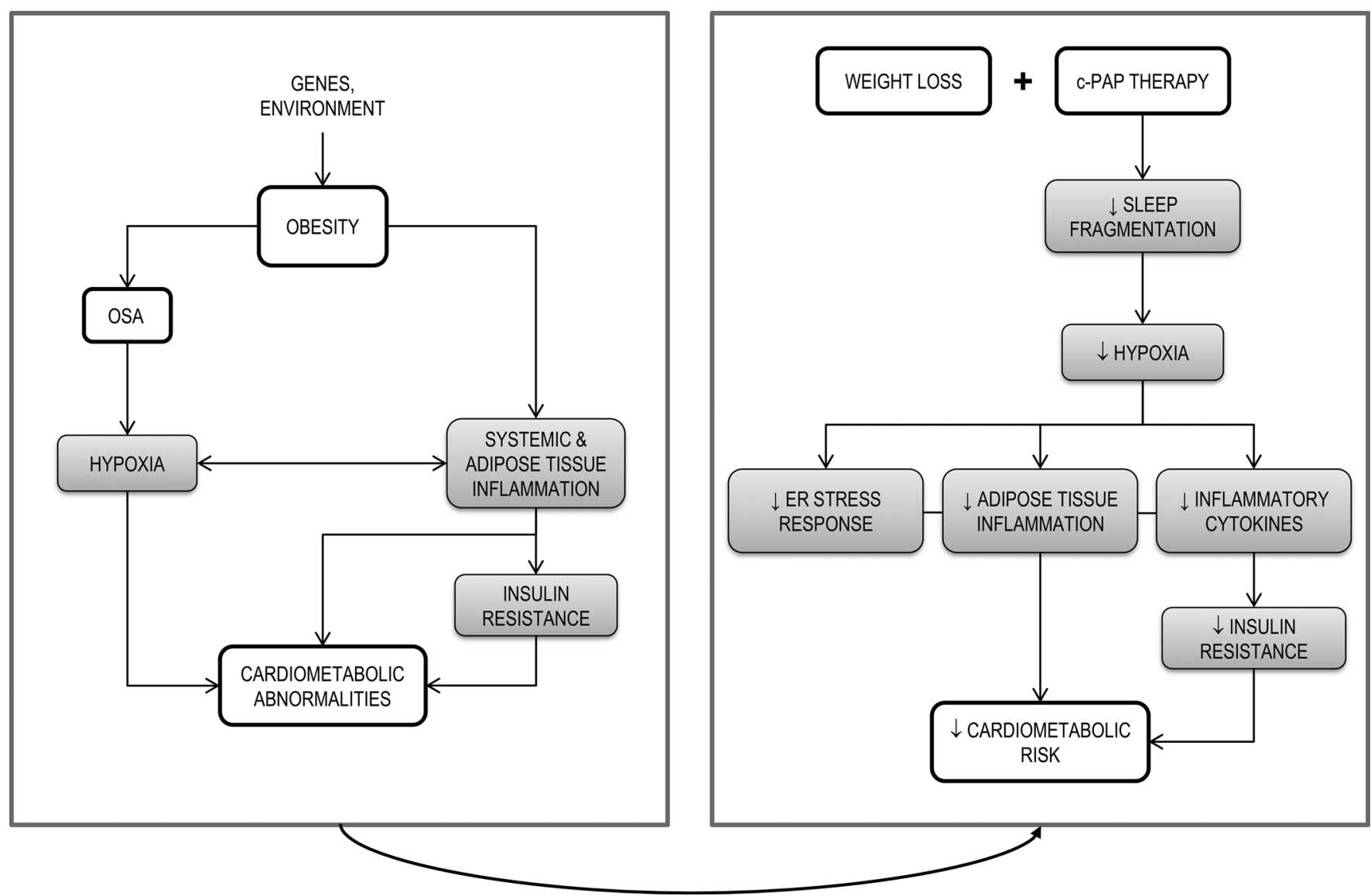

Figure 7. Proposed model of the effects of obesity, OSA, and c-PAP therapy on systemic and adipose tissue inflammation and cardiometabolic abnormalities. OSA, obstructive sleep apnea; c-PAP, continuous positive airway pressure.

and subcutaneous adipose tissue depots $(19,34,45)$. In addition, we did not find sex-related differences in systemic and subcutaneous inflammatory markers among all study groups evaluated (data not shown) but cannot exclude the possibility that, in a larger group of obese subjects with or without OSA, some differences among the experimental groups could be sex related. Finally, the severity of OSA (assessed by measuring the AHI; Table 1) was significantly higher at baseline in therapeutic c-PAP subjects compared with subtherapeutic c-PAP subjects. It is possible that, in these individuals, this could have favored the adherence to c-PAP therapy due to greater awareness of the severity of their disease.

We believe that the strengths of this study include (a) the accurate clinical and metabolic characterization of two matched morbidly obese groups that differed only in the presence or absence of OSA; (b) the simultaneous measurement of serum levels of cytokines, chemokines, growth factors, and subcutaneous adipose tissue ER stress/inflammation gene markers; and (c) the comparative analysis of the endpoints in obese OSA subjects undergoing effective (therapeutic) versus not effective (subtherapeutic) c-PAP therapy.

\section{Methods}

Participants. From February 2012 through December 2015, 31 obese patients affected by moderate-to-severe OSA, who were scheduled to undergo c-PAP therapy, participated in this study and were evaluated at baseline (T0) and after 24 weeks (T1) of therapeutic c-PAP. OSA subjects were compared with 15 BMI- and agematched subjects who had a negative single-night polysomnogram (Figure 1 and Table 1). Obese subjects affected by loudly snoring were evaluated both at the Endocrinology Unit and Respiratory Diseases Unit of Azienda Ospedaliero-Universitaria Policlinico Consorziale, Bari, Italy. Each obese participant in the study underwent recording with a Polysomnography e-Series (Compumedics) for at least 7 hours to determine the presence or severity of obstructive respiratory events across the entire night. The severity of OSA was measured by the AHI. As already specified by Grimaldi et al., subjects were considered to have mild 
OSA if AHI was 5-14, moderate OSA if AHI was 15-29, and severe OSA if AHI was $\geq 30$ (46). No obese subjects had autoimmune inflammatory diseases, cancer, type 2 diabetes, severe kidney or liver diseases, stroke, ischemic or valvular heart disease, obesity hypoventilation syndrome, secondary causes of obesity, or were taking medications that affected body weight, glucose metabolism, and inflammatory markers. All participants had a stable weight in the 4 weeks preceding the screening tests, and, when on blood pressureor lipid-lowering medications, participants had stable blood pressure and lipid values for the preceding 12 weeks. c-PAP machines were provided at no cost by the National Health Service.

Study design and aims. Obese subjects were investigated before (T0) and at the end of the intervention (T1) (Figure 1). The intervention in OSA subjects consisted of a 24-week c-PAP therapy in addition to a weight-loss program. Therapeutic c-PAP intervention was defined as use of the c-PAP mask for $\geq 4$ hours per night. BMI- and age-matched non-OSA obese subjects treated with weight-loss program only served as controls (Figure 1 and Table 1). Furthermore, OSA obese subjects with a c-PAP compliance of less than 4 hours per night (objectively demonstrated by the device software) were considered as an additional control (subtherapeutic c-PAP) group and also included in the analyses (Figure 1 and Table 1).

All participants underwent anthropometric assessment, blood sampling for biochemical measurements, and needle biopsy of subcutaneous adipose tissue from the abdominal region (performed under local anesthesia with $1 \%$ xylocaine, 14-20 cm lateral to the umbilicus) for subsequent gene expression analyses. The fasting blood samples at baseline and 24 weeks later were used for the measurement of glucose, lipid profile, AST, ALT, CRP, and uric acid using Autoanalyzer (Dimension Vista 1500 Lab System, Siemens). Serum insulin was measured using a human insulin radioimmunoassay (Linco). HbA1c was measured using an automated HPLC system (D-10 Hemoglobin Analyzer). ESR was assessed using a fully automated analyzer (Test 1 YDL Alifax, Siemens). Complete blood count was measured by an automated cell counter (ADVIA 2120, Siemens).

The primary aim of the study was to investigate the effects of OSA and c-PAP on changes in adipose tissue inflammatory/hypoxia markers, here assessed by measuring mRNA levels of genes related to macrophage infiltration, hypoxia, and ER stress. Additional endpoints included the effects on systemic inflammatory markers as well as anthropometric, metabolic, and cardiovascular parameters.

To help the participants obtain the necessary skills to achieve a weight loss, all enrolled subjects underwent individual counseling sessions to implement behavioral strategies related to eating and physical activity. Therefore, participants were instructed to modify and reduce their usual daily energy intake by $500 \mathrm{kCa}$ according to the dietician's instructions. Throughout the program, both OSA and non-OSA obese subjects were expected to engage in regular exercise and were instructed to self-monitor the minutes of physical activity and to visit the clinic every month for consultations with a registered dietitian and a physician. Physical activity was assessed using the International Physical Activity Questionnaire (IPAQ) (47). The volume of both physical activity and time spent sitting per week were derived from the IPAQ validity and reliability study and expressed as MET $\times \mathrm{min} / \mathrm{wk}$ and $\mathrm{min} / \mathrm{wk}$, respectively (47).

Titration of c-PAP was performed in the Sleep Disorders and Respiratory Diseases Unit using the 3B RESmart AutoAPAP CPAP Machine. Data were analyzed by two experienced physicians using customized software. Optimal c-PAP pressure used in the active arm of the study was defined as the pressure that abolished all apneas and the patient's snoring. Adherence to c-PAP was defined as c-PAP use for $\geq 4$ hours per night (48). Adherence was assessed by use of a c-PAP device with compliance software.

$R N A$ isolation and quantitative RT-PCR. Total RNA was isolated from abdominal subcutaneous adipose tissue biopsies using the RNeasy Lipid Tissue Mini kit (Qiagen), which has been previously used to achieve optimal lysis of tissues rich in lipids, such as adipose tissue, and purification of high-quality total RNA (49). RNA was quantified by a NanoDrop Spectrophotometer (ND-1000; NanoDrop Technologies). The purity of the RNA was spectrophotometrically assessed by measuring the ratio of absorbance at $260 \mathrm{~nm}$ and 280 nm. 250 ng purified RNA was reverse transcribed with High-Capacity cDNA Synthesis Kits (Applied Biosystems). Primer sequences for each gene (ATF4, CHOP, ERO-1, HIF1 $\alpha$, TLR4, NLRP3, CD68, CD36, IL-6, MCP-1, PDGF $\beta$, VEGF $\alpha$, and GLUT1) are provided in Supplemental Table 1. Transcript levels of genes were quantified by real-time PCR (7300 Real-Time PCR System; Applied Biosystems). cDNA (12.5 ng) was amplified at the following conditions, using the TaqMan Universal PCR Master Mix (Applied Biosystems): $95^{\circ} \mathrm{C}$ for 10 minutes, followed by 39 cycles of 15 seconds at $95^{\circ} \mathrm{C}$ and 1 minute at $60^{\circ} \mathrm{C}$; amplification specificity was confirmed by melting curve analysis. The mRNA level of each target gene was normalized using $18 \mathrm{~S}$ as internal control $(49,50)$. 
Multiplex analysis of cytokine production. Concentrations of various cytokines in blood serum were analyzed as previously described (50), using a multiplex biometrix immunoassay (Bio-Plex Human Cytokine Assay) from Bio-Rad. The following cytokines were assayed: IL-1 $\beta$, IL-1ra, IL-2, IL-4, IL-5, IL-6, IL-7, IL-8, IL-9, IL-10, IL-12 (p70), IL-13, IL-15, IL-17, FGF basic, eotaxin, G-CSF, GM-CSF, IFN- $\gamma$, IP-10, MCP-1 (MCAF), MIP-1 $\alpha$, MIP-1 $\beta$, PDGF $\beta$, RANTES, TNF- $\alpha$, and VEGF $\alpha$. The inter- and intra-assay coefficients of variation for all cytokines under investigation were less than $10 \%$.

Statistics. All participants applied the c-PAP mask for at least 1 hour per night and for $70 \%$ of the nights. Analyses were performed with SPSS software (version 21; IBM). Two-sided $P$ values of less than 0.05 were considered statistically significant. Normally distributed continuous variables were summarized through the use of mean and SD. Nonnormally distributed continuous variables were summarized by the use of median and interquartile range. Categorical variables were summarized by the use of frequency and percentage. Unpaired, two-tailed $t$ tests were used to compare the difference between non-OSA and OSA groups at baseline. One-sample, two-tailed $t$ test was used to compare the change from baseline in endpoints of interest for each group. One-way ANOVA and Tukey's post-hoc tests were used to compare the difference in the change in endpoints of interest from baseline between the three groups.

Study approval. The study protocol was approved by the independent ethics committee of the Azienda Ospedaliero Universitaria Policlinico Consorziale, Bari, Italy (approval 152/2012). All subjects gave written informed consent prior to the inclusion in the study.

\section{Author contributions}

S. Perrini initiated the development of and conceived the study, designed the experiments, performed the adipose tissue biopsies, was responsible for recruiting and characterization of obese subjects, collected and analyzed the results, and discussed and wrote the manuscript. A. Cignarelli helped to recruit obese subjects, provided expertise in the interpretation of serum cytokine and adipose tissue gene expression data, performed statistical analysis, and discussed the manuscript. VNQ, VAF, PC, and OR were responsible for recruiting and characterization of OSA obese subjects, performed and analyzed the single-night polysomnogram in all enrolled obese subjects, and discussed the manuscript. SK, A. Ciavarella, and MB helped with recruiting and characterization of the obese subjects and collected and analyzed all clinical data of enrolled subjects. RF, S. Porro, PN, and VAG performed serum cytokine and gene expression analyses and collected and analyzed the data. AN and LL conceived the study, contributed to the interpretation of the data, and discussed the article. FG conceived the study, designed the experiments, coordinated and directed the project, edited and revised the manuscript. FG is the guarantor of this work and, as such, had full access to all the data in the study and takes responsibility for the integrity of the data and the accuracy of the data analyses. All authors contributed to the interpretation of the data.

\section{Acknowledgments}

The authors are grateful to all volunteers who participated to the study. Funding was provided by Ministero dell'Università e della Ricerca, Italy, and Progetti di Rilevante Interesse Nazionale.

Address correspondence to: Francesco Giorgino, Department of Emergency and Organ Transplantation - Section of Internal Medicine, Endocrinology, Andrology and Metabolic Diseases, University of Bari Aldo Moro, Piazza Giulio Cesare, 11, I-70124 Bari, Italy. Phone 39.080.5478689; Email: francesco.giorgino@uniba.it.

1. Hotamisligil GS. Inflammation and metabolic disorders. Nature. 2006;444(7121):860-867.

2. Dewan NA, Nieto FJ, Somers VK. Intermittent hypoxemia and OSA: implications for comorbidities. Chest. 2015;147(1):266-274.

3. Garvey JF, Pengo MF, Drakatos P, Kent BD. Epidemiological aspects of obstructive sleep apnea. J Thorac Dis. 2015;7(5):920929.

4. Drager LF, Li J, Reinke C, Bevans-Fonti S, Jun JC, Polotsky VY. Intermittent hypoxia exacerbates metabolic effects of diet-induced obesity. Obesity (Silver Spring). 2011;19(11):2167-2174.

5. Yadav R, et al. Impairment of high-density lipoprotein resistance to lipid peroxidation and adipose tissue inflammation in obesity complicated by obstructive sleep apnea. J Clin Endocrinol Metab. 2014;99(9):3390-3398.

6. Nadeem R, et al. Serum inflammatory markers in obstructive sleep apnea: a meta-analysis. J Clin Sleep Med. 2013;9(10):1003-1012.

7. Trayhurn P. Hypoxia and adipose tissue function and dysfunction in obesity. Physiol Rev. 2013;93(1):1-21.

8. Pasarica M, et al. Reduced adipose tissue oxygenation in human obesity: evidence for rarefaction, macrophage chemotaxis, and inflammation without an angiogenic response. Diabetes. 2009;58(3):718-725.

9. Poulain L, et al. Visceral white fat remodelling contributes to intermittent hypoxia-induced atherogenesis. Eur Respir J. 
2014;43(2):513-522.

10. Hotamisligil GS. Endoplasmic reticulum stress and the inflammatory basis of metabolic disease. Cell. 2010;140(6):900-917.

11. Feng Y, Zhang Z, Dong ZZ. Effects of continuous positive airway pressure therapy on glycaemic control, insulin sensitivity and body mass index in patients with obstructive sleep apnoea and type 2 diabetes: a systematic review and meta-analysis. NPJ Prim Care Respir Med. 2015;25:15005.

12. Xie X, Pan L, Ren D, Du C, Guo Y. Effects of continuous positive airway pressure therapy on systemic inflammation in obstructive sleep apnea: a meta-analysis. Sleep Med. 2013;14(11):1139-1150.

13. Chirinos JA, et al. CPAP, weight loss, or both for obstructive sleep apnea. $N$ Engl J Med. 2014;370(24):2265-2275.

14. Sun K, Kusminski CM, Scherer PE. Adipose tissue remodeling and obesity. J Clin Invest. 2011;121(6):2094-2101.

15. Rausch ME, Weisberg S, Vardhana P, Tortoriello DV. Obesity in C57BL/6J mice is characterized by adipose tissue hypoxia and cytotoxic T-cell infiltration. Int J Obes (Lond). 2008;32(3):451-463.

16. Spencer M, et al. Adipose tissue extracellular matrix and vascular abnormalities in obesity and insulin resistance. J Clin Endocrinol Metab. 2011;96(12):E1990-E1998.

17. O'Rourke RW, et al. Hypoxia-induced inflammatory cytokine secretion in human adipose tissue stromovascular cells. Diabetologia. 2011;54(6):1480-1490.

18. Goossens $\mathrm{GH}$, et al. Increased adipose tissue oxygen tension in obese compared with lean men is accompanied by insulin resistance, impaired adipose tissue capillarization, and inflammation. Circulation. 2011;124(1):67-76.

19. Aron-Wisnewsky J, et al. Chronic intermittent hypoxia is a major trigger for non-alcoholic fatty liver disease in morbid obese. J Hepatol. 2012;56(1):225-233.

20. Reinke C, Bevans-Fonti S, Drager LF, Shin MK, Polotsky VY. Effects of different acute hypoxic regimens on tissue oxygen profiles and metabolic outcomes. J Appl Physiol. 2011;111(3):881-890.

21. Olson NC, et al. Circulating levels of TNF- $\alpha$ are associated with impaired glucose tolerance, increased insulin resistance, and ethnicity: the Insulin Resistance Atherosclerosis Study. J Clin Endocrinol Metab. 2012;97(3):1032-1040.

22. Dixon JB, et al. Surgical vs conventional therapy for weight loss treatment of obstructive sleep apnea: a randomized controlled trial. JAMA. 2012;308(11):1142-1149.

23. Colish J, et al. Obstructive sleep apnea: effects of continuous positive airway pressure on cardiac remodeling as assessed by cardiac biomarkers, echocardiography, and cardiac MRI. Chest. 2012;141(3):674-681.

24. Ishida $\mathrm{K}$, et al. Appropriate use of nasal continuous positive airway pressure decreases elevated C-reactive protein in patients with obstructive sleep apnea. Chest. 2009;136(1):125-129.

25. Patruno V, et al. Fixed and autoadjusting continuous positive airway pressure treatments are not similar in reducing cardiovascular risk factors in patients with obstructive sleep apnea. Chest. 2007;131(5):1393-1399.

26. Akashiba T, Akahoshi T, Kawahara S, Majima T, Horie T. Effects of long-term nasal continuous positive airway pressure on C-reactive protein in patients with obstructive sleep apnea syndrome. Intern Med. 2005;44(8):899-900.

27. Kohler M, et al. Effects of continuous positive airway pressure on systemic inflammation in patients with moderate to severe obstructive sleep apnoea: a randomised controlled trial. Thorax. 2009;64(1):67-73.

28. Kaczmarek E, et al. Molecular biomarkers of vascular dysfunction in obstructive sleep apnea. PLoS ONE. 2013;8(7):e70559.

29. Findley CM, Mitchell RG, Duscha BD, Annex BH, Kontos CD. Plasma levels of soluble Tie2 and vascular endothelial growth factor distinguish critical limb ischemia from intermittent claudication in patients with peripheral arterial disease. J Am Coll Cardiol. 2008;52(5):387-393.

30. Fontana L, Meyer TE, Klein S, Holloszy JO. Long-term calorie restriction is highly effective in reducing the risk for atherosclerosis in humans. Proc Natl Acad Sci USA. 2004;101(17):6659-6663.

31. Cancello R, et al. Reduction of macrophage infiltration and chemoattractant gene expression changes in white adipose tissue of morbidly obese subjects after surgery-induced weight loss. Diabetes. 2005;54(8):2277-2286.

32. Kanda H, et al. MCP-1 contributes to macrophage infiltration into adipose tissue, insulin resistance, and hepatic steatosis in obesity. J Clin Invest. 2006;116(6):1494-1505.

33. Odegaard JI, et al. Macrophage-specific PPARgamma controls alternative activation and improves insulin resistance. Nature. 2007;447(7148):1116-1120.

34. Huber J, et al. CC chemokine and CC chemokine receptor profiles in visceral and subcutaneous adipose tissue are altered in human obesity. J Clin Endocrinol Metab. 2008;93(8):3215-3221.

35. Weisberg SP, McCann D, Desai M, Rosenbaum M, Leibel RL, Ferrante AW. Obesity is associated with macrophage accumulation in adipose tissue. J Clin Invest. 2003;112(12):1796-1808.

36. Lumeng CN, Deyoung SM, Bodzin JL, Saltiel AR. Increased inflammatory properties of adipose tissue macrophages recruited during diet-induced obesity. Diabetes. 2007;56(1):16-23.

37. Yang Z, Ming XF. CD36: the common soil for inflammation in obesity and atherosclerosis? Cardiovasc Res. 2011;89(3):485-486.

38. Kennedy DJ, Kuchibhotla S, Westfall KM, Silverstein RL, Morton RE, Febbraio M. A CD36-dependent pathway enhances macrophage and adipose tissue inflammation and impairs insulin signalling. Cardiovasc Res. 2011;89(3):604-613.

39. Huang H, Jing G, Wang JJ, Sheibani N, Zhang SX. ATF4 is a novel regulator of MCP-1 in microvascular endothelial cells J Inflamm (Lond). 2015;12:31.

40. Lerner AG, et al. IRE1 $\alpha$ induces thioredoxin-interacting protein to activate the NLRP3 inflammasome and promote programmed cell death under irremediable ER stress. Cell Metab. 2012;16(2):250-264.

41. Oslowski CM, et al. Thioredoxin-interacting protein mediates ER stress-induced $\beta$ cell death through initiation of the inflammasome. Cell Metab. 2012;16(2):265-273.

42. Bronner DN, et al. Endoplasmic reticulum stress activates the inflammasome via NLRP3- and caspase-2-driven mitochondrial damage. Immunity. 2015;43(3):451-462.

43. Magkos F, et al. Effects of moderate and subsequent progressive weight loss on metabolic function and adipose tissue biology in humans with obesity. Cell Metab. 2016;23(4):591-601.

44. Kursawe R, et al. A role of the inflammasome in the low storage capacity of the abdominal subcutaneous adipose tissue in obese adolescents. Diabetes. 2016;65(3):610-618. 
45. Harman-Boehm I, et al. Macrophage infiltration into omental versus subcutaneous fat across different populations: effect of regional adiposity and the comorbidities of obesity. J Clin Endocrinol Metab. 2007;92(6):2240-2247.

46. Grimaldi D, Beccuti G, Touma C, Van Cauter E, Mokhlesi B. Association of obstructive sleep apnea in rapid eye movement sleep with reduced glycemic control in type 2 diabetes: therapeutic implications. Diabetes Care. 2014;37(2):355-363.

47. Craig CL, et al. International physical activity questionnaire: 12-country reliability and validity. Med Sci Sports Exerc. 2003;35(8):1381-1395.

48. Sawyer AM, Gooneratne NS, Marcus CL, Ofer D, Richards KC, Weaver TE. A systematic review of CPAP adherence across age groups: clinical and empiric insights for developing CPAP adherence interventions. Sleep Med Rev. 2011;15(6):343-356.

49. Perrini S, et al. Fat depot-related differences in gene expression, adiponectin secretion, and insulin action and signalling in human adipocytes differentiated in vitro from precursor stromal cells. Diabetologia. 2008;51(1):155-164.

50. Perrini S, et al. Differences in gene expression and cytokine release profiles highlight the heterogeneity of distinct subsets of adipose tissue-derived stem cells in the subcutaneous and visceral adipose tissue in humans. PLoS One. 2013;8(3):e57892. 